\title{
SYNCHRONOUS SOLUTIONS OF POWER SYSTEMS: EXISTENCE, UNIQUENESS AND STABILITY*
}

\author{
BY \\ SHERWIN J. SKAR \\ Iowa State University
}

\begin{abstract}
This paper contains a study of the asymptotic stability and uniqueness of equilibrium solutions of multi-dimensional Hamiltonian-like systems. The results are applied to the swing equations, the classical model for power systems. By developing some results in matrix theory, it is shown that asymptotically stable equilibrium solutions may exist even though most rotor angle pairs are more than $90^{\circ}$, some even $180^{\circ}$, out of phase. In contrast to the numerical criteria usually used, an analytic criterion for the existence of asymptotically stable equilibrium solutions of the swing equations is given.
\end{abstract}

1. Introduction. Most authors studying the stability of the swing equations for power systems have investigated only those equilibrium solutions for which all the rotor angles are less than $90^{\circ}$ out of phase with each other. In this paper we develop examples of swing equations for which asymptotically stable equilibrium solutions exist even though most of the rotor angle differences are greater than $90^{\circ}$ in magnitude; some such differences may be $180^{\circ}$.

It is the usual procedure to determine the existence of a post-fault equilibrium solution by numerical calculations. In this paper we give an analytic criterion for the existence of stable equilibrium solutions.

In general we study the stability, instability, uniqueness and existence of equilibrium solutions of the swing equations. The first three properties are studied by investigating the following system which is a model for many physical systems, including the swing equations:

$$
\dot{y}=-A y-g(z), \quad \dot{z}=B y,
$$

where $y \in R^{k}, z \in R^{l}, k+l=m, x=\left(y^{T}, z^{T}\right)^{T} \in R^{m}, A$ and $B$ are constant real $k \times k$ and $l \times k$ matrices respectively, and $g: R^{l} \rightarrow R^{k}$ is a continuous map. For the swing equations $k \neq l$.

When conservative forces are involved, system (1) will be a damped Hamiltonian-like system. In Sec. 2 we discuss the stability and uniqueness of equilibrium points of such systems. In Sec. 3 we apply these results to the swing equations and prove the existence result.

\footnotetext{
* Received September 29, 1979; revised version received January 1, 1980. This research was supported by the National Science Foundation under Grant ENG 77-28446.
} 
In Sec. 4 we develop some matrix theory which will allow us to find swing equations of arbitrarily large dimension for which stable equilibrium solutions exist even though most of the rotor angle differences are greater than $90^{\circ}$ in magnitude.

Most of the notation used is standard and will be used without explanation. The symbols $A^{T}$ and $A^{-1}$ will denote the transpose and inverse, respectively, of a matrix $A=\left(a_{i j}\right)$ whose $(i-j)$ th element is $a_{i j}$. All vectors $x$ are column vectors; hence $x^{T}$ is a row vector. The constant vectors $y^{*} \in R^{k}, z^{*} \in R^{l}$ are an equilibrium point of system (1) if $A y^{*}+g\left(z^{*}\right)=0$ and $B y^{*}=0$. Let $I_{n}$ be the $n \times n$ identity matrix. Let $\partial g / \partial z$ be the Jacobian matrix of $g$.

A submatrix of $A$ is a matrix formed by eliminating from $A$ certain rows and columns. A principal submatrix of $A$ is a matrix formed by eliminating a row from $A$ if and only if the corresponding column is eliminated. We use several characterizations for the positive definiteness of a symmetric matrix (see [8], p. 306).

For a real-valued function $h(y, z)$ with $y \in R^{k}, z \in R^{l}$ we let

$$
\nabla_{y} h=\left(\partial h / \partial y_{1}, \ldots, \partial h / \partial y_{k}\right)^{T}, \quad \nabla_{z} h=\left(\partial h / \partial z_{1}, \ldots, \partial h / \partial z_{l}\right)^{T} .
$$

2. Hamiltonian-like systems. In this section we study the stability of damped Hamiltonian-like systems of the form (1).

2.1 Definition. We say that a system of ordinary differential equations is Hamiltonianlike if it has the form

$$
\dot{y}=-L \nabla_{z} h(y, z), \quad \dot{z}=L^{T} \nabla_{y} h(y, z)
$$

where $y \in R^{k}, z \in R^{l}, h$ is a differentiable real-valued function of $y$ and $z$, and $L$ is a $k \times l$ real matrix.

The function $h$ is an integral of system (2) and system (2) is Hamiltonian in form if $k=l$ and $L$ is the identity matrix. System (2) is called damped if a linear term $-A y$ is added to the right-hand side of Eq. (2a).

In the following theorem if we let $L=K^{-1} B^{T}$ and $h(y, z)=V\left(x, z^{*}\right)$ (see Eq. (5) below), then system (1) is a damped Hamiltonian-like system. One example of such a system with $k \neq l$ is the swing equations with zero transfer conductances (see Sec. 3).

The following theorem lists the results for Hamiltonian-like forms of system (1).

2.2 Theorem. Let $A, B$, and $g$ be defined as in Eq. (1). Let $K$ be a symmetric positive definite $k \times k$ matrix. Let $f(z)$ be a real-valued function of $l$ variables with continuous second partial derivatives. Suppose that $K A+A^{T} K$ is positive definite, that $B$ has rank $l$ with $l \leq k$, and that

$$
g(z)=K^{-1} B^{T} \nabla_{z} f(z)
$$

Define the Hessian matrix

$$
J(z)=\left(\frac{\partial^{2} f}{\partial z_{i} \partial z_{j}}\right) .
$$

(a) If $x^{*}=\left(y^{* T}, z^{* T}\right)^{T}$ is an equilibrium point of system (1), then $y^{*}=0$ and $g\left(z^{*}\right)=0$.

(b) Suppose that $g\left(z^{*}\right)=0$ for some $z^{*} \in R^{l}$ and that $J\left(z^{*}\right)$ is positive definite. Then $x^{*}=\left(0^{T}, z^{* T}\right)^{T}$ is an asymptotically stable equilibrium point of system (1). 
(c) Suppose that $g\left(z^{*}\right)=0$ for some $z^{*} \in R^{l}$ and that $J\left(z^{*}\right)$ is not positive semidefinite. Then $x^{*}=\left(0^{T}, z^{* T}\right)^{T}$ is not an asymptotically stable equilibrium point of system (1). If in addition $x^{*}$ is an isolated equilibrium point of (1), then $x^{*}$ is not stable.

(d) Let $g\left(z^{*}\right)=0$ for some $z^{*} \in R^{l}$ and let $S$ be a convex subset of $R^{l}$ with $z^{*} \in S$. Suppose that $J(z)$ is positive definite and that Eq. (3) holds for all $z \in S$. Then $x^{*}=\left(0^{T}, z^{* T}\right)^{T}$ is the only equilibrium point of system (1) in $R^{k} \times S$.

Proof. (a) Suppose that $x^{*}=\left(y^{* T}, z^{* T}\right)^{T}$ is an equilibrium point of system (1). Then $A y^{*}+g\left(z^{*}\right)=0$ and $B y^{*}=0$. Thus

$$
\frac{1}{2} y^{* T}\left(A^{T} K+K A\right) y^{*}=y^{* T} K\left(A y^{*}\right)=-y^{* T} K g\left(z^{*}\right)=-y^{* T} B^{T} \nabla_{z} f(z)=0 .
$$

Since $A^{T} K+K A$ is positive definite, $y^{*}=0$.

For parts (b) and (c), define

$$
V\left(x ; z^{*}\right)=\frac{1}{2} y^{T} K y+f(z)-f\left(z^{*}\right)
$$

where $z^{*} \in R^{l}$ is fixed, $g\left(z^{*}\right)=0$, and $x=\left(y^{T}, z^{T}\right)^{T} \in R^{m}$. The derivative of $V$ along solutions of system (1) is

$$
\frac{d V}{d t}=y^{T} K[-A y-g(z)]+\left[\nabla_{z} f(z)\right]^{T} B y=-\frac{1}{2} y^{T}\left(K A+A^{T} K\right) y \leq 0 .
$$

Define

$$
G=\left\{x \in R^{m}: d V / d t=0\right\}=\left\{x=\left(y^{T}, z^{T}\right)^{T}: z \in R^{l}, y \in R^{k}, y=0\right\} .
$$

By inspection of system (1), the only solutions of (1) which remain in $G$ for all $t$ are the constant solutions or equilibrium points. Define the Hessian matrix

$$
H=\left.\left(\frac{\partial^{2} V}{\partial x_{i} \partial x_{j}}\right)\right|_{x=x^{*}}=\left[\begin{array}{ll}
K & 0 \\
0 & J\left(z^{*}\right)
\end{array}\right] .
$$

Since $K$ is positive definite, $H$ is positive definite if and only if $J\left(z^{*}\right)$ is.

Because $B$ has full rank and $K^{-1} B^{T} \nabla_{z} f\left(z^{*}\right)=g\left(z^{*}\right)=0$, we know that $\nabla_{z} f\left(z^{*}\right)=0$. Hence $V\left(x^{*} ; z^{*}\right)=0$ and

$$
\left.\nabla_{x} V\right|_{x=x^{*}}=\left[\nabla_{z}^{K y^{*}} f\left(z^{*}\right)\right]=0,
$$

where $x^{*}=\left(0^{T}, z^{* T}\right)$. Thus $x^{*}$ is a zero and a critical point of $V$.

(b) Suppose that $J\left(z^{*}\right)$ and hence $H$ is positive definite. Then $x^{*}$ is a relative minimum point for $V\left(x ; z^{*}\right)$ (see [2], p. 376).

Since det $J\left(z^{*}\right) \neq 0, \nabla_{z} f$ is one-to-one in a neighborhood of $z^{*}$ by the inverse function theorem (see [2], p. 372). Hence, $g(z)$ is one-to-one in that neighborhood of $z^{*}$ because $K^{-1}$ and $B^{T}$ are one-to-one. Thus, there is a neighborhood $N$ of $x^{*}$ such that for $x \in N$ we have $V\left(x ; z^{*}\right)>0$ if $x \neq x^{*}$ and $g(z)$ is one-to-one if $\left(y^{T}, z^{T}\right)^{T}=x \in N$.

Therefore $x^{*}$ can be the only equilibrium point and hence the only invariant subset of $G \cap N$. By the invariance theorem $[4,9] x^{*}$ is an asymptotically stable equilibrium point of system (1).

(c) Suppose that $J\left(z^{*}\right)$ and hence $H$ is not positive semi-definite. Then $x^{*}$ is a saddle point for $V\left(x ; z^{*}\right)$ (see [2]). Hence, there is an open region $M \subset R^{m}$ containing $x^{*}$ on its boundary such that $V\left(x ; z^{*}\right)<0$ for $x \in M$. Since $d V / d t \leq 0$, a solution starting in $M$ cannot approach $x^{*}$ as $t$ increases. Thus $x^{*}$ is not an asymptotically stable equilibrium point of system (1). 
Suppose that, in addition, $x^{*}$ is an isolated equilibrium point. Let $N$ be a bounded neighborhood of $x^{*}$ containing no other equilibrium points of (1). By invariance theory (see [4], p. 203), a solution starting in $M \cap N$ must either leave the set $N$ or approach an invariant subset of $G$. Since $\left\{x^{*}\right\}$ is the only invariant subset of $G$ in $N$, the point $x^{*}$ cannot be a stable equilibrium point.

(d) By part (a) we need only to show that $g(z)$ is one-to-one on $S$.

Let $z^{1}$ and $z^{2}$ be two distinct points in $S$. Define $z(u)=u z^{2}+(1-u) z^{1}$ for $u \in R$ and $w=z^{2}-z^{1}$. Then $z(u) \in S$ for $0 \leq u \leq 1$ by convexity of $S$ and $d z / d u=w$. Let $J(u)=$ $J(z(u))$ and

$$
q(u)=\left\{\nabla_{z} f[z(u)]-\nabla_{z} f\left(z^{1}\right)\right\}^{T} w .
$$

Then

$$
\frac{d q}{d u}=\frac{d}{d u}\left(\sum_{i=1}^{l}\left\{\frac{\partial f}{\partial z_{i}}[z(u)]-\frac{\partial f}{\partial z_{i}}\left(z^{1}\right) \mid w_{i}\right)=\sum_{i=1}^{l} \sum_{j=1}^{l} \frac{\partial^{2} f}{\partial z_{i} \partial z_{j}} w_{i} w_{j}=w^{T} J(u) w .\right.
$$

Since $J(u)$ is positive definite for $0 \leq u \leq 1, d q / d u>0$ for $0<u<1$. Hence $0=q(0)<q(1)$.

If $\nabla_{z} f\left(z^{1}\right)=\nabla_{z} f\left(z^{2}\right)$, then $q(1)=0$, which is a contradiction. Since $B^{T}$ and $K^{-1}$ are one-to-one, $g\left(z^{1}\right) \neq g\left(z^{2}\right)$.

If system (1) is damped Hamiltonian in form, then we can apply Theorem 2.2 with $k=l$ and $B=K$.

3. Power systems. The problem of transient stability of systems of synchronous generators modeled by the "swing equations" has been studied since the beginning of the electric age $[1,5]$. In recent years Lyapunov theory has been applied to the problem $[7,11]$.

In this section we show that the swing equations take the form of system (1) so that we can apply the results of Sec. 2 . The Lyapunov function used in this section is equivalent to a classical Lyapunov function used in the study of power systems. Derivatives of this Lyapunov function along solutions are not negative definite, so that asymptotic stability does not follow directly from the classical Lyapunov theorems. By applying the invariance theorem we can conclude the asymptotic stability of the equilibrium solutions with a negative semi-definite derivative. ${ }^{*}$ We also give results on existence and uniqueness of stable equilibrium solutions.

In the classical model for power systems with negligible transfer conductances, a set of $n$ interconnected synchronous generators is assumed to be governed by the system of differential equations (see $[7,11])$

$$
M_{i} \ddot{\delta}_{i}+d_{i} \dot{\delta}_{i}=Q_{i}-E_{i}^{2} G_{i i}-\sum_{\substack{j=1 \\ j \neq i}}^{n} E_{i} E_{j} Y_{i j} \sin \left(\delta_{i}-\delta_{j}\right)
$$

for $i=1, \ldots, n$. For the $i$ th generator $\delta_{i}$ is the rotor angle, $M_{i}>0$ is the moment of inertia, $d_{i}>0$ is a damping constant, $Q_{i}$ is the mechanical power, and the remaining parameters have technical meanings as discussed in the literature (see $[7,11])$. All parameters except $\delta_{i}$ are assumed to be constant during the transient.

* Professor R. K. Miller suggested the application of invariance theory to the swing equation. 
3.1 Definition. A solution $\left(\delta_{i}, \ldots, \delta_{n}\right)$ is called a synchronous solution of system (6) if $\delta_{i}=\omega_{0}$ is constant for $i=1, \ldots, n$.

A principal goal of a power systems engineer is to ensure that a system of synchronous generators approaches a synchronous solution.

If we assume that $\left(\delta_{1}, \ldots, \delta_{n}\right)$ is a synchronous solution of system (6) and we add together all $n$ equations, we find that

Hence, we define

$$
\sum_{i=1}^{n} d_{i} \grave{\delta}_{i}=\sum_{i=1}^{n}\left(Q_{i}-E_{i}^{2} G_{i i}\right)
$$

$$
\omega_{0}=\left[\sum_{i=1}^{n}\left(Q_{i}-E_{i}^{2} G_{i i}\right)\right] /\left(\sum_{i=1}^{n} d_{i}\right)
$$

to be consistent with Definition 3.1.

For convenience we define

$$
P_{i j}=P_{j i}=E_{i} E_{j} Y_{i j}, \quad P_{i}=Q_{i}-E_{i}^{2} G_{i i}-d_{i} \omega_{0} .
$$

Then $\sum_{i=1}^{n} P_{i}=0$. We make the change of variables

$$
\begin{aligned}
& \omega_{i}=\delta_{i}-\omega_{0} \text { for } i=1, \ldots, n, \quad \sigma_{i}=\delta_{i}-\delta_{n} \text { for } i=1, \ldots, n-1, \\
& \sigma_{n}=0, \quad \rho=\delta_{n} .
\end{aligned}
$$

In the new variables and parameters system (6) takes the form

$$
\begin{aligned}
M_{i} \dot{\omega}_{i} & =-d_{i} \omega_{i}+P_{i}-\sum_{\substack{j=1 \\
j \neq i}}^{n} P_{i j} \sin \left(\sigma_{i}-\sigma_{j}\right) \quad i=1, \ldots, n, \\
\dot{\sigma}_{i} & =\omega_{i}-\omega_{n} \text { for } i=1, \ldots, n-1, \\
\dot{\rho} & =\omega_{n}+\omega_{0},
\end{aligned}
$$

where $\sum_{i=1}^{n} P_{i}=0$ and $\sigma_{n}=0$. By introducing the extra parameter $\sigma_{n}=0$ we can write system (8) in a more compact form than otherwise.

For the remainder of the paper we will call system (8) the swing equations. The system of equations (8a), (8b), and (9) is equivalent to system (6). However, system (8) is independent of $\rho$; solutions of system (6) may be found from solutions of system (8) by the integration indicated by Eq. (9). Hence, we will focus our attention on system (8).

For convenience we define

$$
\sigma=\left(\sigma_{1}, \ldots, \sigma_{n-1}\right)^{T} \text { and } \omega=\left(\omega_{1}, \ldots, \omega_{n}\right)^{T} .
$$

3.2 Remark. A synchronous solution of system (6) corresponds to an equilibrium point $\omega^{*}, \sigma^{*}$ of system (8) with the property that $\omega^{*}=0 \in R^{n}$. We see this by examining the two systems and considering the discussion after Definition 3.1.

Many authors study only stable equilibrium points which occur in the set

$$
S=\left\{\sigma \in R^{n-1}:\left|\sigma_{i}\right|<\frac{\pi}{2},\left|\sigma_{i}-\sigma_{j}\right|<\frac{\pi}{2} \text { for } i, j=1, \ldots, n-1, i \neq j\right\} .
$$

We see that $S$ is convex and that if $\sigma \in S$, then $\cos \left(\sigma_{i}-\sigma_{j}\right)>0$ for $i, j=1, \ldots, n$ (recall $\sigma_{n}=0$ ). We will see that any equilibrium point $\omega^{*}, \sigma^{*}$ with $\sigma^{*} \in S$ will be asymptotically stable, a well-known result. However, in Sec. 4 we will see that $\sigma^{*}$ may be far outside $S$ and still be an asymptotically stable equilibrium point. 
3.3 Remark. If the parameters of system (1) are defined in the following way, then system (1) and system (8) are equivalent.

Let $k=n, l=n-1$. Let $y=\omega, z=\sigma$ and $\sigma_{n}=z_{n}=0$. Define

$$
A=\operatorname{diag}\left(d_{i} / M_{i}\right)
$$

and let $B=\left(b_{i j}\right)$ be an $(n-1) \times n$ matrix where

$$
\begin{aligned}
& b_{i j}=1 \quad \text { if } \quad i=j=1, \ldots, n-1 \\
& =-1 \quad \text { if } j=n, \quad i=1, \ldots, n-1 \\
& =0 \quad \text { if } i \neq j ; \quad i, j=1, \ldots, n-1 \text {. }
\end{aligned}
$$

For $i=1, \ldots, n$ let

$$
g_{i}(z)=\frac{1}{M_{i}}\left[-P_{i}+\sum_{\substack{j=1 \\ j \neq i}}^{n} P_{i j} \sin \left(z_{i}-z_{j}\right)\right]
$$

and $g(z)=\left(g_{1}(z), \ldots, g_{n}(z)\right)^{T}$.

Since systems (1) and (8) are equivalent through the definitions of Remark 3.3, we may apply the results of Sec. 2 to system (8). To do so we define

$$
\begin{aligned}
p_{i}(\sigma) & =M_{i} g_{i}(\sigma) \\
& =-P_{i}+P_{i n} \sin \sigma_{i}+\sum_{\substack{j=1 \\
j \neq i}}^{n-1} P_{i j} \sin \left(\sigma_{i}-\sigma_{j}\right)
\end{aligned}
$$

for $i=1, \ldots, n$. We let

$$
p(\sigma)=\left[p_{1}(\sigma), \ldots, p_{n-1}(\sigma)\right]^{T} \in R^{n-1} .
$$

The following result is a direct application of Theorem 2.2. It provides sufficient conditions for the asymptotic stability and the instability of the swing equations (system (8)). It will be used in Sec. 4 to find stable equilibrium points of system (8) for which most of the rotor angles are more than $90^{\circ}$ out of phase. Unlike many previous authors, we are able to conclude asymptotic stability rather than only stability. The result is almost a necessary and sufficient condition for stability of an equilibrium point. The critical case for which $\partial p / \partial \sigma$ is positive semidefinite and singular is the only case not treated in the following theorem. The theorem also contains a uniqueness result.

3.4 Theorem. Suppose that $\omega^{*}, \sigma^{*}$ is an equilibrium point of system (8). Then $\omega^{*}=0$ and system (8) is equivalent to a Hamiltonian-like form of system (1). Let $p$ be defined as in Eq. (13).

(a) If the Jacobian matrix $\left.(\partial p / \partial \sigma)\right|_{\sigma=\sigma^{*}}$ is positive definite, then $\omega^{*}, \sigma^{*}$ is an asymptotically stable equilibrium point of system (8). If $\sigma^{*} \in S$ (see Eq. (10)), then $\left.(\partial p / \partial \sigma)\right|_{\sigma=\sigma^{*}}$ is positive definite.

(b) If the Jacobian matrix $\left.(\partial p / \partial \sigma)\right|_{\sigma=\sigma^{*}}$ is not positive semi-definite, then $\omega^{*}, \sigma^{*}$ is not asymptotically stable equilibrium point of system (8). If, in addition, $\sigma^{*}$ is an isolated solution of $p(\sigma)=0$, then $\omega^{*}, \sigma^{*}$ is not a stable equilibrium point of system (8).

(c) Suppose that $T$ is a convex subset of $R^{n-1}$ such that if $\sigma \in T$, then $\partial p / \partial \sigma$ is positive definite. In particular, we may set $T=S$ where $S$ is defined in Eq. (10). Then $p(\sigma)$ is one-to-one on $T$, and $\omega^{*}, \sigma^{*}$ is the only equilibrium point of system (8) with $\sigma^{*} \in T$. 
Proof. Let $A, B$, and $g$ be defined in Remark 3.3. We apply Theorem 2.2 to system (8) with

$$
f(z)=\left[-\sum_{i=1}^{n-1}\left(P_{i} z_{i}+P_{i n} \cos z_{i}\right)-\sum_{i=2}^{n-1} \sum_{j=1}^{i-1} P_{i j} \cos \left(z_{i}-z_{j}\right)\right]
$$

and with $K=M=\operatorname{diag}\left(M_{i}\right)$. We see that $p(\sigma)=p(z)=\nabla_{z} f(z)$. In a straightforward way we see that the hypotheses of Theorem 2.2 are satisfied and $\partial p /\left.\partial \sigma\right|_{\sigma^{*}}=J\left(\sigma^{*}\right)=J\left(z^{*}\right)$. If $\sigma \in S$, then we see that $J(\sigma)$ is symmetric and diagonally dominant so that $J(\sigma)$ is positive definite.

The region $S$ is not the largest region containing the origin in which $\partial p / \partial \sigma$ is positive definite. If we assume a conjecture of Tavora and Smith [10] that each connected region in which $\partial p / \partial \sigma$ is positive definite is convex, then system (8) has at most one equilibrium point in any such region. The author was unable to follow the uniqueness proof of Tavora and Smith.

The existence of an equilibrium point for the swing equations is usually discovered in practice by numerical methods. The next result gives an analytic criterion for the existence of a stable equilibrium point for the swing equations.

3.5 THEOREM. Referring to system (8), suppose that

$$
\sum_{i=1}^{n-1} \frac{P_{i}^{2}}{P_{i n}^{2}}<1
$$

Then there exists a unique vector $\sigma^{*} \in S$ (see Eq. (10)) such that $\omega^{*}=0, \sigma^{*}$ is an asymptotically stable equilibrium point of system (8).

Proof. Let $p$ be defined in Eq. (13). By Theorem 3.4, $p$ is one-to-one on $S$. Let $P=\left(P_{1}, \ldots, P_{n-1}\right)^{T} \in R^{n-1}$ and $Q(\sigma)=p(\sigma)+P$. Then $Q$ is one-to-one on $S$. Let $T=Q(S)$. Define $U=\left\{q \in R^{n-1}: \sum_{i=1}^{n-1}\left(q_{i}^{2} / P_{i n}^{2}\right)<1\right\}$.

If $U \subset T$, then we define $\sigma^{*}=Q^{-1}(P) \in Q^{-1}(T)=S$ so that $p\left(\sigma^{*}\right)=Q\left(\sigma^{*}\right)-P=0$. By Theorem $3.4, w^{*}=0, \sigma^{*}$ is asymptotically stable.

To show that $U \subset T$ we show that if $q$ is on the boundary of $T$, then $\sum_{i=1}^{n-1}\left(q_{i}^{2} / P_{i n}^{2}\right) \geq 1$ so that $q \notin U$. But $Q(0)=0 \in U \cap T$ so that $U \subset T$. Now, $q$ is a boundary point of $T$ if and only if $q=Q(\sigma)$ for some $\sigma$ on the boundary of $S$. The point $\sigma$ is on the boundary of $S$ if and only if $\sigma_{k}=\pi / 2, \sigma_{k}=-\pi / 2$, or $\sigma_{k}-\sigma_{l}=\pi / 2$ for some $k$, $l \in\{1, \ldots, n-1\}$. We see that if $\sigma$ is on the boundary of $S$, then $\cos \left(\sigma_{i}-\sigma_{j}\right) \geq 0$ and $\cos \sigma_{i} \geq 0$ for all $i, j=1, \ldots, n-1$ with $i \neq j$. We consider three cases.

(i) Suppose that $\sigma_{k}=\pi / 2$ where $k \in\{1, \ldots, n-1\}$. Let $q=Q(\sigma)$. Then since $\sin \left(\pi / 2-\sigma_{i}\right)=\cos \sigma_{i} \geq 0$ for $i=1, \ldots, n-1$, we have that

$$
\begin{aligned}
\frac{q_{k}^{2}}{P_{k n}^{2}}=\frac{1}{P_{k n}^{2}}\left[\sum_{\substack{i=1 \\
i \neq k}}^{n-1} \sum_{\substack{j=1 \\
j \neq k}}^{n-1} P_{k i} P_{k j} \sin \left(\sigma_{k}-\sigma_{i}\right) \sin \left(\sigma_{k}-\sigma_{j}\right)\right. \\
\left.+2 \sum_{\substack{i=1 \\
i \neq k}}^{n-1} P_{k i} P_{k n} \sin \left(\sigma_{k}-\sigma_{i}\right) \sin \sigma_{k}+P_{k n}^{2} \sin ^{2} \sigma_{k}\right] \\
=\left[\sum_{\substack{i=1 \\
i \neq k}}^{n-1} \sum_{\substack{j=1 \\
j \neq k}}^{n-1} \frac{P_{k i} P_{k j}}{P_{k n}^{2}} \cos \sigma_{i} \cos \sigma_{j}+2 \sum_{\substack{i=1 \\
i \neq k}}^{n-1} \frac{P_{k i}}{P_{k n}} \cos \sigma_{i}+1\right] \geq 1 .
\end{aligned}
$$


Hence

$$
\sum_{i=1}^{n-1}\left(q_{i}^{2} / P_{i n}^{2}\right) \geq 1 .
$$

(ii) Suppose that $\sigma_{k}=-\pi / 2$ where $k \in\{1, \ldots, n-1\}$. We repeat the argument of (i), noticing that $\sin \left(-\pi / 2-\sigma_{i}\right)=-\cos \sigma_{i} \leq 0$ for $i=1, \ldots, n-1$. The negative signs cancel to give the same result.

(iii) Suppose that $\sigma_{k}=\sigma_{l}+\pi / 2$ for some $k, l \in\{1, \ldots, n-1\}$ with $k \neq l$. Let $q=Q(\sigma)$. For $i=1, \ldots, n-1$ we make the substitutions $\sin \left(\sigma_{k}-\sigma_{i}\right)=\cos \left(\sigma_{l}-\sigma_{i}\right) \geq 0$, $\sin \left(\sigma_{l}-\sigma_{i}\right)=-\cos \left(\sigma_{k}-\sigma_{i}\right) \leq 0$, $\sin \sigma_{k}=\cos \sigma_{l} \geq 0$, and $\sin \sigma_{l}=-\cos \sigma_{k} \leq 0$ in the equations $q_{k}=Q_{k}(\sigma)$ and $q_{l}=Q_{l}(\sigma)$ to show that

$$
q_{k}^{2} / P_{k n}^{2}+q_{l}^{2} / P_{l n}^{2} \geq 1 .
$$

Hence, (14) is true for all $q$ on the boundary of $T$. Q.E.D.

By looking at limiting cases in which some of the $P_{i j}$ are zero and for which (14) becomes an equation, we see that Theorem 3.5 is "best possible" for this kind of criterion. We note that because the numbering of the machines is arbitrary, the theorem is actually more general.

4. Stable examples. Most studies of the Lyapunov stability of power systems discuss only equilibrium points for which the rotor angles are less than $90^{\circ}$ out of phase with each other; that is, equilibrium points $\omega^{*}, \sigma^{*}$ for which $\sigma^{*} \in S$ (see Eq. (10)). It is known (see [10]) that some equilibrium points outside $S$ may be stable, a fact which may be established by a continuity argument. However, it appears to be often assumed that stable equilibrium points occur in or near the region $S$.

In this section we will see that stable equilibrium points for swing equations may occur far outside the region $S$. In fact, there are examples of swing equations which have a stable equilibrium point for which a majority of the rotor angles are more than $90^{\circ}$ out of phase; some may be $180^{\circ}$ out of phase.

To find such examples we will develop some conditions for positive definiteness and apply Theorem 3.4. We see from Eq. (13) that

$$
\begin{aligned}
\frac{\partial p_{i}}{\partial \sigma_{j}} & =P_{i n} \cos \sigma_{i}+\sum_{\substack{r=1 \\
r \neq i}}^{n-1} P_{i r} \cos \left(\sigma_{i}-\sigma_{r}\right) \quad \text { if } \quad i=j \\
& =-P_{i j} \cos \left(\sigma_{i}-\sigma_{j}\right) \quad \text { if } \quad i \neq j .
\end{aligned}
$$

We will begin the section with a somewhat lengthy but necessary search for a criterion based on the signs of the numbers $P_{i j} \cos \left(\sigma_{i}-\sigma_{j}\right)$ to determine whether $\partial p / \partial \sigma$ can be positive definite if the $P_{i j}>0$ are chosen properly. Using this criterion, we will choose the vector $\sigma^{*}$ so that the signs of $\cos \left(\sigma_{i}^{*}-\sigma_{j}^{*}\right)$ allow $\left.(\partial p / \partial \sigma)\right|_{\sigma=\sigma^{*}}$ to be positive definite for some choices of $P_{i j}$. We will then choose the $P_{i}$ so that $\omega^{*}=0, \sigma^{*}$ is an equilibrium point (and by Theorem 3.4 asymptotically stable) of system (8).

The following definitions will be useful. We assume that $H=\left(h_{i j}\right)$ is a real $n \times n$ symmetric matrix (such as $\partial p / \partial \sigma$ ). 
4.1 Definition. Let

$$
\begin{aligned}
& d_{i j}=-h_{i j} \text { for } i, j=1, \ldots, n \text { with } i \neq j \\
& d_{i i}=0 \text { for } i=1, \ldots, n+1 \\
& d_{0 i}=d_{i 0}=\sum_{j=1}^{n} h_{i j} \text { for } i=1, \ldots, n .
\end{aligned}
$$

The matrix $D(H)=\left(d_{i j}\right)$ is an $(n+1) \times(n+1)$ matrix called the configuration matrix for $H$. Note that $d_{i j}=d_{j i}$.

4.2 Definition. Let $D(H)=\left(d_{i j}\right)$ be the configuration matrix for $H$. Define a matrix $Q(H)=\left(q_{i j}\right)$ such that

$$
\begin{aligned}
& q_{i j}=-1 \quad \text { if } \quad d_{i j}<0 \\
& =0 \text { if } d_{i j}=0 \\
& =1 \text { if } d_{i j}>0 \text {. }
\end{aligned}
$$

The matrix $Q(H)$ is called the sign configuration matrix. Two symmetric matrices $H$ and $J$ have the same sign configuration if $Q(J)=Q(H)$.

As we will see, the next two definitions are criteria for positive definiteness (or lack thereof). One is the negation of the other.

4.3 Definition. Let $D(H)=\left(d_{i j}\right)$. We say that $H$ has property $R$ if there exists an ordered $(n+1)$-tuple $k=\left(k_{0}, \ldots, k_{n}\right)$ such that $k$ is a permutation of elements of $\{0, \ldots, n\}$ and such that for all $i=1, \ldots, n$ it is true that $d_{j l}>0$ with $l=k_{i}$ for some $j \in\left\{k_{0}, \ldots, k_{i-1}\right\}$.

For any $T \subset\{0, \ldots, n\}$ we let $\bar{T}=\{i \in\{0, \ldots, n\}: i \notin T\}$.

4.4 Definition. Let $D(H)=\left(d_{i j}\right)$. We say that $H$ has property $N$ if there exists a proper subset $T$ of $\{0, \ldots, n\}$ such that $d_{i j} \leq 0$ whenever $i \in T$ and $j \in \bar{T}$.

4.5 Lemma. Let $H$ be a real $n \times n$ symmetric matrix. Then $H$ has property $R$ if and only if $H$ does not have property $N$.

Proof. If we assume $N$ and $R$, we can show by induction that the subset $T$ assumed in $N$ cannot be proper. If we assume that $H$ does not have property $N$, then we can construct the $(n+1)$-tuple of $R$ by induction.

4.6 Lemma. Let $H$ be a real $n \times n$ symmetric matrix with $D(H)=\left(d_{i j}\right)$. If there exists $k \in\{0, \ldots, n\}$ such that $d_{k j} \leq 0$ for all $j \in\{0, \ldots, n\}$, then $H$ is not positive definite.

Proof. If $k=(1, \ldots, n)$, then a diagonal element of $H$ is not positive. If $k=0$, we form a new matrix $H^{\prime}$ by adding all the rows of $H$ to its first row and all the columns of $H$ to the first column. Although this transformation preserves positive definiteness, the first diagonal element of $H^{\prime}$ is not positive.

4.7 Lemma. Let $H$ be a real $n \times n$ symmetric matrix with $D(H)=\left(d_{i j}\right)$ such that $d_{i j} \geq 0$ for $i, j=0, \ldots, n$. If some principal submatrix $F$ of $H$ has property $N$, then $H$ has property $N$.

Proof. Let $F$ be the $r \times r$ submatrix formed from $H$ by eliminating the rows and columns indexed by the elements of a proper subset $P^{\prime}$ of $\{1, \ldots, n\}$. Let $\bar{P}=\{0\} \cup P^{\prime}$. Let $P$ and $\bar{P}$ be disjoint sets whose union is $\{0, \ldots, n\}$. By hypothesis we may form a proper 
subset $T$ of $\{0, \ldots, r\}$ which satisfies the criterion of property $N$ for $F$. Let $T^{\prime}$ be the set of integers $l \in P$ such that row $l$ of $H$ corresponds to row $j$ of $F$ for some $j \in T$. Then $T^{\prime}$ satisfies the criterion of property $N$ for $H$.

4.8 Lemma. Let $H$ be a real $n \times n$ symmetric matrix with $D(H)=\left(d_{i j}\right)$.

(a) If $H$ has property $N$, then $H$ is not positive definite.

(b) If $d_{i j}>0$ for $i, j=0, \ldots, n$ with $i \neq j$, then $H$ is positive definite.

(c) Suppose that $d_{i j} \geq 0$ for $i, j=0, \ldots, n$. Then $H$ is positive definite if and only if $H$ has property $R$.

(d) If $H$ has property $R$, then there exists a real $n \times n$ symmetric matrix $H^{\prime}$ with the same sign configuration as $H$ such that $H^{\prime}$ is positive definite.

(e) If $d_{i j}<0$ for some $i, j \in\{0, \ldots, n\}$ then there exists a real $n \times n$ symmetric matrix $H^{\prime}$ with the same sign configuration as $H$ such that $H^{\prime}$ is not positive definite.

Proof. (a) Let $T$ be the subset given in property $N$ for $H$. Let $F$ be the submatrix of $H$ formed by eliminating the rows and columns with indices in $\bar{T}$. By Lemma 4.6 we see that $F$, and hence $H$, is not positive definite.

Part (b) follows from part (c).

(c) We see that one direction follows from part (a). We prove the other direction by induction. The result for $2 \times 2$ matrices follows by an easy calculation.

Assume that (c) is true for all $(n-1) \times(n-1)$ matrices. Let $H$ be the $n \times n$ matrix of the hypothesis. Since $H$ does not have property $N$, there exists an integer $l$ with $1 \leq l \leq n$ such that $d_{0}>0$. Without loss of generality let $l=n$. Let

$$
H=\left[\begin{array}{ll}
A & b \\
b^{T} & c
\end{array}\right]
$$

where

$$
b^{T}=\left(h_{i n}, \ldots, h_{n-1, n}\right)=\left(-d_{i n}, \ldots,-d_{n-1, n}\right), \quad c=h_{n n}=\sum_{i=0}^{n} d_{i n},
$$

and $A$ is the principal submatrix of $H$ formed by eliminating the last row and column of $H$. By Lemmas 4.5 and 4.7 , the submatrix $A$ has property $R$, so that $A$ is positive definite by the induction hypothesis. Let $A=\left(a_{i j}\right)$ and $A^{-1}=\left(\hat{a}_{i j}\right)$. Since $A$ is an $M$-matrix (see [3] or [6]), we know that $\hat{a}_{i j} \geq 0$ for all $i, j=1, \ldots, n$.

Let $e=(1,1, \ldots, 1)^{T} \in R^{n-1}$. Then the $i$ th component of $A e$ is

$$
(A e)_{i}=\sum_{j=1}^{n-1} h_{i j}=d_{i n}+d_{i 0} \geq d_{i n} .
$$

Thus for $j=1, \ldots, n-1$

$$
1=e_{j}=\left(A^{-1} A e\right)_{j}=\sum_{i=1}^{n-1} \hat{a}_{j i}(A e)_{i} \geq \sum_{i=1}^{n-1} \hat{a}_{j i} d_{i n} .
$$

Since $A$ is positive definite, $H$ is positive definite if and only if det $H>0$. By a result from matrix theory (see [8], p. 45)

$$
\operatorname{det} H=\left(c-b^{T} A^{-1} b\right) \operatorname{det} A .
$$


But

$$
\begin{aligned}
c-b^{T} A^{-1} b & =\sum_{i=0}^{n} d_{i n}-\sum_{i=1}^{n-1} \sum_{j=1}^{n-1}\left(-d_{i n}\right) \hat{a}_{i j}\left(-d_{j n}\right) \\
& =d_{0 n}+\sum_{i=1}^{n-1} d_{i n}\left(1-\sum_{j=1}^{n-1} \hat{a}_{i j} d_{j n}\right) \\
& \geq d_{0 n}>0 .
\end{aligned}
$$

Hence det $H>0$, so that $H$ is positive definite.

(d) Assume that $H$ has property $R$ with $D(H)=\left(d_{i j}\right)$. Let $f_{i j}=d_{i j}$ if $d_{i j} \geq 0$ and $f_{i j}=\varepsilon d_{i j}$ for $\varepsilon>0$ if $d_{i j} \leq 0$. Define $\bar{H}(\varepsilon)$ as $D(\bar{H}(\varepsilon))=\left(f_{i j}(\varepsilon)\right)$. Then $\bar{H}(0)$ is positive definite by part (c). By continuity of eigenvalues there exists $\varepsilon>0$ such that $\bar{H}(\varepsilon)$ is positive definite, but clearly $\bar{H}(\varepsilon)$ has the same sign configuration as $H$ for any $\varepsilon>0$.

(e) For this part we choose $d_{i j}<0$ so small that

$$
h_{i i}=\sum_{l=0}^{n} d_{i l}<0 .
$$

We may use Lemma 4.8 to show that many examples of the swing equations exist with asymptotically stable equilibrium points far outside the region usually studied.

4.9 ThEOREM. Let $\sigma^{*} \in R^{n-1}$. Suppose that there is an $n$-tuple $k=\left(k_{1}, \ldots, k_{n}\right)$ such that $k$ is a permutation of elements of $\{1, \ldots, n\}$ and such that for all $i=2, \ldots, n$ it is true that $\cos \left(\sigma_{j}^{*}-\sigma_{l}^{*}\right)>0$ if $l=k_{i}$ for some $j \in\left\{k_{1}, \ldots, k_{i-1}\right\}$. (Recall $\sigma_{n}^{*}=0$.) Then we may choose real numbers $P_{i}$ and $P_{i j}=P_{j i}>0$ for $i, j=1, \ldots, n$ with $i \neq j$ such that $\omega^{*}=0$, $\sigma^{*}$ is an asymptotically stable equilibrium point of system (8) for any $M_{i}>0$ and $d_{i}>0$, $i=1, \ldots, n$.

Proof. Let $p(\sigma)$ be defined in Eq. (13). Let $H=\left.(\partial p / \partial \sigma)\right|_{\sigma=\sigma^{*}}$ and $D(H)=\left(d_{i j}\right)$ (see Definition 4.1). Then $d_{i j}=P_{i j} \cos \left(\sigma_{i}^{*}-\sigma_{j}^{*}\right)$ for all $i, j=1, \ldots, n-1$ with $i \neq j$ and $d_{i 0}=P_{\text {in }} \cos \sigma_{i}^{*}$ for $i=1, \ldots, n-1$. The hypothesis determines that $H$ has property $R$ for any choices of the $P_{i j}$. By Lemma 4.8(d) we may choose the $P_{i j}$ so that $H$ is positive definite. With these choices of $P_{i j}$ we choose the $P_{i}$ so that $\omega^{*}=0, \sigma^{*}$ is an equilibrium point of system (8).

In applying the previous theorem we are restricted in the possible sign configurations of $\partial p / \partial \sigma$ by the fact that the right-hand side of Eq. (8a) is periodic in each component of $\sigma$. Hence, if $\omega^{*}=0, \sigma^{*}$ is an equilibrium point of system (8), then we may assume without loss of generality that $\left|\sigma_{i}^{*}\right| \leq \pi$ for $i=1, \ldots, n-1$. This assumption restricts the possible choices of $\sigma^{*}$ for which $\cos \left(\sigma_{i}^{*}-\sigma_{j}^{*}\right) \leq 0$. Except in low dimensions, for all $\sigma^{*}$ many of the numbers $\cos \left(\sigma_{i}^{*}-\sigma_{j}^{*}\right)$ are positive. Nevertheless, examples of stable equilibrium points exist for which most of the numbers $\cos \left(\sigma_{i}^{*}-\sigma_{j}^{*}\right)$ are negative.

4.10 Example. Let $n=3 l+3$ for some positive integer $l$. Let

$$
\sigma_{1}^{*}=\pi / 3, \quad \sigma_{2}^{*}=-\pi / 3, \quad \sigma_{2+i}^{*}=0, \quad \sigma_{2+l+i}^{*}=2 \pi / 3, \quad \sigma_{2+2 l+i}^{*}=-2 \pi / 3
$$

for $i=1, \ldots, l$. With $k=(1, \ldots, n)$ we apply Theorem 4.9 to see that $\omega^{*}=0, \sigma^{*}$ is an asymptotically stable equilibrium point of system (8) for some choices of parameters. Of the numbers $\cos \sigma_{i}^{*}$ a total of $l+2$ are positive and $2 l$ are negative. Of the numbers $\cos \left(\sigma_{i}^{*}-\sigma_{j}^{*}\right)$ a total of $\left(3 l^{2}+5 l\right) / 2$ are positive and $\left(6 l^{2}+4 l+2\right) / 2$ are negative. For 
large $l$ there are about twice as many negative as positive numbers of this kind; that is, the associated synchronous solution is attractive even though most of the rotor angles are more than $90^{\circ}$ out of phase with each other.

4.11 Example. As a specific example we define the parameters of system (8) as follows: $n=4, d_{i}>0, M_{i}>0$ for $i=1,2,3,4$, and

$$
\begin{aligned}
P_{1} & =11 \sqrt{ } 3 / 2=-P_{4}, & P_{2}=\sqrt{ } 3 / 2=-P_{3}, \\
P_{12}=10, & P_{13}=1, & P_{14}=1, \quad P_{23}=10, \quad P_{24}=1, \quad P_{34}=10 .
\end{aligned}
$$

This system has an asymptotically stable equilibrium point $\omega^{*}=0, \sigma^{*}$ with

$$
\begin{aligned}
\sigma_{1}^{*} & =\pi, \quad \sigma_{2}^{*}=2 \pi / 3, \quad \sigma_{3}^{*}=\pi / 3 \\
\sigma_{1}^{*}-\sigma_{2}^{*} & =\pi / 3, \quad \sigma_{1}^{*}-\sigma_{3}^{*}=2 \pi / 3, \quad \sigma_{2}^{*}-\sigma_{3}^{*}=\pi / 3 .
\end{aligned}
$$

In this example

$$
\left.\frac{\partial p}{\partial \sigma}\right|_{\sigma=\sigma^{*}}=\frac{1}{2}\left[\begin{array}{crc}
7 & -10 & 1 \\
-10 & 19 & -10 \\
1 & -10 & 19
\end{array}\right]
$$

which is positive definite, so that $\omega^{*}, \sigma^{*}$ is asymptotically stable by Theorem $3.4(\mathrm{a})$. Thus, a synchronous solution may be attractive even though some of the rotor angles are $180^{\circ}$ out of phase.

\section{REFERENCES}

[1] P. M. Anderson and A. A. Fouad, Power system control and stability, Iowa State University Press, Ames, 1977

[2] T. M. Apostal, Mathematical analysis, second edition, Addison-Wesley, Reading, Mass., 1975

[3] M. Araki, M-matrices (matrices with nonpositive off-diagonal elements and positive principal minors), Publication 74/19, Imperial College of Science and Technology, London, 1974

[4] F. Brauer and J. A. Nohel, The qualitative theory of ordinary differential equations, W. A. Benjamin, New York, 1969

[5] O. G. C. Dahl, Electric power circuits: theory and applications, Vol. II, Power system stability, McGrawHill, New York, 1938

[6] M. Fiedler and V. Pták, On matrices with nonpositive off-diagonal elements and positive principal minors, Czech. Math. J., 12, 382-400 (1962)

[7] A. A. Fouad, Stability theory - criteria for transient stability, ERDA Report, Engineering Research Institute, Iowa State University, Ames, 1975

[8] F. R. Gantmacher, The theory of matrices, Vol. I, Chelsea, New York, 1959

[9] W. Hahn, Stability of motion, Springer-Verlag, New York, 1967

[10] C. J. Tavora and O. J. M. Smith, Equilibrium analysis of power systems, IEEE Trans. Power Apparatus and Systems, 91, 1131-1137 (1972)

[11] J. L. Willems, Direct methods for transient stability studies in power systems analysis, IEEE Trans. Automat. Contr., 16, 332-341 (1971) 